\title{
Rheumatoid Arthritis-Associated Interstitial Lung Disease: Diagnostic Dilemma
}

\author{
Mark J. Hamblin and Maureen R. Horton \\ Division of Pulmonary and Critical Care Medicine, Johns Hopkins University School of Medicine, 1830 East Monument Street, \\ 5th Floor, Baltimore, MD 21205, USA \\ Correspondence should be addressed to Maureen R. Horton, mhorton2@jhmi.edu
}

Received 21 October 2010; Revised 1 March 2011; Accepted 24 March 2011

Academic Editor: D. Bouros

Copyright ( 2011 M. J. Hamblin and M. R. Horton. This is an open access article distributed under the Creative Commons Attribution License, which permits unrestricted use, distribution, and reproduction in any medium, provided the original work is properly cited.

\begin{abstract}
Interstitial lung disease (ILD) is an increasingly recognized complication of rheumatoid arthritis (RA) contributing to significantly increased morbidity and mortality. Diagnosis can be challenging since patients are unlikely to report dyspnea due to an overall decrease in physical activity with advanced arthritic symptoms. Additionally, infections, drug toxicity, and environmental toxins can mimic ILD, creating significant diagnostic dilemmas for the clinician. In this paper we will explore an effective clinical algorithm for the diagnosis of RA-ILD. We will also discuss features of drug-related toxicities, infections, and environmental toxins that comprise the main entities in the differential diagnosis of RA-ILD. Finally, we will explore the known and experimental treatment options that may have some benefit in the treatment of RA-ILD.
\end{abstract}

\section{Introduction}

It has been estimated that nearly $50 \%$ of rheumatoid arthritis (RA) patients will develop some form of respiratory abnormality during their lifetime $[1,2]$. In fact, one study by Toyoshima et al. placed pulmonary disease as the second leading cause of death in RA accounting for nearly $20 \%$ of the mortality [3]. While there are a myriad of pulmonary complications associated with RA (Table 1), the most debilitating remains rheumatoid arthritis-associated interstitial lung disease (RA-ILD). Advancements in imaging technology have improved our ability to diagnose RAILD, and what once was thought to be a relatively rare complication is now suspected to affect $20-30 \%$ of RA patients [4].

In this paper, we will discuss the initial evaluation of suspected RA-ILD focusing on screening algorithms and radiologic features of the disease. However, screening and diagnosis of RA-ILD is only one aspect of care. Once the presence of RA-ILD is certain, the challenge for clinicians comes in distinguishing an exacerbation of RA-ILD from infection, drug toxicity, or a host of other comorbid disease entities. We will explore a practical approach to the workup and treatment of a clinical deterioration in the established RA-ILD patient, and the following case history will serve as a framework for this discussion highlighting some of the inherent challenges.

\section{Case Discussion}

A 64-year-old woman was originally diagnosed with RA in 1986, and over the next several years her treatment regimen escalated from nonsteroidal anti-inflammatory medications with gold salts to D-penicillamine and methotrexate (MTX). In 2000, she was started on etanercept. Her arthritis symptoms were manageable on a regimen of etanercept and nonsteroidal anti-inflammatory medications, but after a few years of therapy she began to develop a mild non-productive cough but no noticeable dyspnea on exertion. In 2007, she was admitted to the hospital with acute renal failure. A kidney biopsy showed focal and segmental sclerosis. A chest X-ray from that hospitalization was abnormal, and a high-resolution chest CT (HRCT) demonstrated increased reticular markings consistent with an interstitial lung disease (Figure 1). She was started on peritoneal dialysis and 
TABLE 1: Respiratory complications of rheumatoid arthritis.

\begin{tabular}{|c|c|}
\hline Lung structure & Disease manifestations \\
\hline \multirow{11}{*}{ Lung parenchyma } & Interstitial lung disease (ILD) \\
\hline & Usual interstitial pneumonitis (UIP) \\
\hline & Nonspecific interstitial pneumonitis (NSIP) \\
\hline & Bronchiolitis obliterans with organizing pneumonia (BOOP) \\
\hline & Lymphocytic interstitial pneumonitis (LIP) \\
\hline & Desquamative interstitial pneumonitis (DIP) \\
\hline & Diffuse alveolar damage (DAD) \\
\hline & Drug-induced pneumonitis \\
\hline & Rheumatoid nodules (necrobiotic nodules) \\
\hline & Caplan's syndrome (silicosis associated with RA) \\
\hline & Infectious complications \\
\hline \multirow{4}{*}{ Airways } & Chronic obstructive pulmonary disease (COPD) \\
\hline & Bullous emphysema \\
\hline & Bronchiectasis \\
\hline & Obliterative bronchiolitis (Constrictive bronchiolitis) \\
\hline \multirow{3}{*}{ Pleura } & Pleuritis \\
\hline & Pleural effusion \\
\hline & Spontaneous pneumothorax \\
\hline \multirow{2}{*}{ Vascular } & Pulmonary hypertension \\
\hline & Diffuse alveolar hemorrhage \\
\hline \multirow{2}{*}{ Extrapulmonary } & Diaphragm weakness \\
\hline & Cricoarytenoid arthritis with extrathoracic obstruction \\
\hline
\end{tabular}

prednisone for the renal failure but did not undergo any further evaluation of her pulmonary infiltrates.

Her cough persisted, and she eventually became dyspneic climbing a flight of stairs. She was finally referred for an evaluation by a pulmonologist. Additional findings obtained at her initial visit included a history of myocardial infarction in 2001 and hypothyroidism treated with levothyroxine. She also kept two cockatiels as pets. She had no other hobbies or environmental exposures, and she was never a smoker. In addition to her cough, dyspnea, and arthritis pain, she also admitted night sweats over the past 6 months, but no weight loss. Her exam revealed a low-grade fever at $99.6 \mathrm{~F}$, and she had bibasilar crackles but no digital clubbing. Her oxygen saturation was $100 \%$ at rest. Pulmonary function tests (PFTs) were obtained which demonstrated a preserved total lung capacity and forced vital capacity at 106\% and 90\% of predicted, respectively, but her diffusing capacity (DLCO) was moderately reduced at $61 \%$ of predicted.

In summary, this was a woman with long-standing RA greatly increasing her risk for RA-ILD. However, she also had a history of using several medications (gold salts, D-penicillamine, methotrexate and etanercept) all of which have been associated with drug-induced interstitial pneumonitis. In addition, she had exposure to cockatiels that could induce an unrelated hypersensitivity pneumonitis. Her low-grade fevers and night sweats in the setting of chronic immunosuppression could also suggest a possible chronic infectious etiology or even an occult malignancy. Finally, she had a history of a myocardial infarction and renal failure, and she was now on peritoneal dialysis. She had no record of an echocardiogram since 2001, so a possible coexistent medical problem could account for her symptoms of dyspnea and cough.

\section{Establishing a Diagnosis of RA-ILD}

Although our index case is representative of a standard referral to our practice, the majority of cases of RAILD are uncovered following patient complaints to their primary care physician or rheumatologist regarding the onset of progressive dyspnea on exertion. A growing number of rheumatology practices are facilitating a standardized screening algorithm for RA-ILD; however, the most costeffective approach to screening has not been universally established [5].

In the era prior to the routine use of HRCT, the prevalence of RA-ILD was about $1-5 \%$ based on clinical exam and chest X-ray $[6,7]$. In contrast, histological analysis of lung tissue from RA patients found the presence of pulmonary interstitial abnormalities in roughly $80 \%$ of patients [8]. A more recent clinical study employing HRCT also detected abnormalities in $80 \%$ of patients evaluated for dyspnea; however, only about $25 \%$ of these changes were consistent with ILD [9]. Additional findings included bronchiectasis, bronchiolitis, and pulmonary nodules. Similarly, other studies utilizing HRCT also found that only about $20-30 \%$ of symptomatic patients had ground glass 


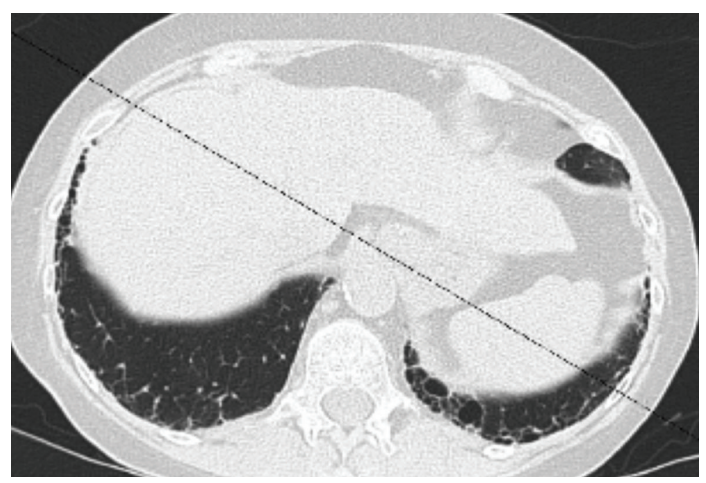

(a)

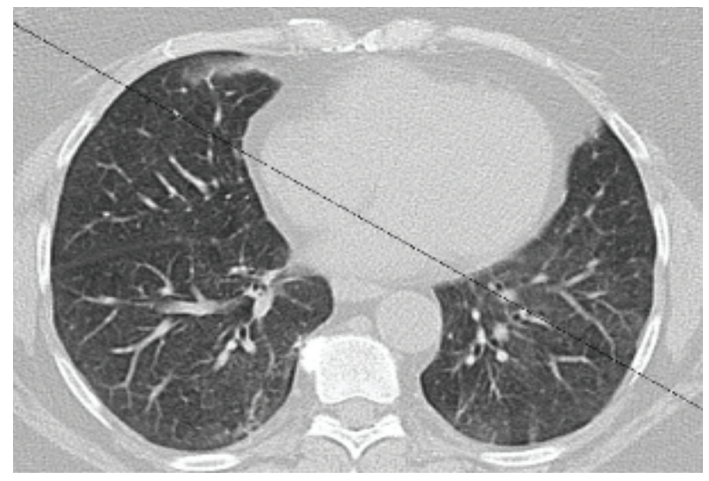

(b)

FIGURE 1: HRCT demonstrating predominantly reticular findings (a) as well as ground glass changes (b).

opacities or increased reticular markings consistent with ILD $[4,10]$. Given the poor sensitivity of chest X-ray and the high prevalence of abnormalities that do not always correlate to clinical disease on HRCT, we do not recommend imaging as an initial screening tool in asymptomatic patients.

Pulmonary function testing, and more specifically the DLCO, appears to be the most sensitive test available to screen for the presence of RA-ILD. Similar to our index case, Dawson et al. found that $80 \%$ of patients with RA had a reduced DLCO, while only $5-15 \%$ of patients had a purely restrictive defect on spirometry [10]. While the DLCO may be highly sensitive for RA-ILD, the specificity is lacking due to the prevalence of emphysema that can destroy vascular beds thus affecting the DLCO. In fact, Geddes et al. reported obstructive spirometry findings in more than $80 \%$ of RA patients in the form of bronchiectasis, constrictive bronchiolitis, or emphysematous changes [11]. These obstructive disorders also appear to be at least partially the effect of the disease process since obstructive airways disease in a nonsmoking RA population was much higher than a nonsmoking control population [12]. Additionally about $25 \%$ of patients have cricoarytenoid joint involvement, and although it is usually asymptomatic, it can evolve, sclerosing the joint leading to hoarseness, pain, and upper airway obstructive respiratory symptoms [13].
Despite the fact that emphysema alone may decrease the DLCO in the absence of RA-ILD, we still recommend followup imaging when the DLCO is reduced to less than $70 \%$ of predicted, especially in the absence of other abnormalities in the PFTs. This is based on the increasing recognition of "pseudo-normal PFTs" created by combined pulmonary fibrosis and emphysema in the idiopathic pulmonary fibrosis (IPF) population [14]. In several studies, up to $40 \%$ of patients had radiographic evidence of emphysema and pulmonary fibrosis on HRCT. However, they had FVC and TLC values that were normal or only had a borderline restrictive defect, and the FEV1/FVC ratio was often normal or only mildly reduced even with radiographic evidence of severe emphysema [14-16]. In this subset of patients, there was a uniform decrease in the DLCO (less than $40 \%$ in most studies) that was often out of proportion to the rest of the PFT abnormalities. Considering that there is a high prevalence of tobacco use in the RA population, this pattern of combined pulmonary fibrosis and emphysema may be just as common as that seen in IPF; however, further studies are needed [17].

Unfortunately, routine pulmonary function tests on every RA patient have not been established as a cost-effective approach to screening for pulmonary disease [18]. Considering that a number of patients will have abnormalities that do not correlate with clinical disease, we believe that the history and physical remain the vanguards of clinical evaluation until further studies of costeffectiveness are presented [11]. We advocate that each clinic visit include a brief inquiry into the presence of cough or mild dyspnea when climbing a flight of stairs or on an incline, as well as clinical examination for pulmonary crackles or digital clubbing although these are usually only found in advanced disease [19]. Additional patient factors that should increase the suspicion for RAILD include sex, anti-CCP levels, and smoking status. In epidemiologic studies, men are much more likely than women to develop RA-ILD [20]. High levels of anti-CCP antibodies have been associated with pulmonary fibrosis, and tobacco abuse ( $>25$ pack years) may have the strongest association with the development of RA-ILD with an odds ratio of $3.8[21,22]$.

In summary, if dyspnea or cough is present, a complete set of pulmonary tests is indicated including spirometry, lung volumes, and DLCO (Figure 2). A DLCO reduced to less than $70 \%$ of predicted should be the threshold for further imaging with HRCT.

\section{Radiologic Evaluation and Histopathology}

The presence of bibasilar, symmetrical, ground glass infiltrates, increased reticular markings, or honeycombing on HRCT is highly suggestive of ILD [23]. There can be a host of other findings including pleural involvement, rheumatoid nodules, bronchiectasis, emphysema, or even bronchiolitis with or without consolidation [24, 25]. In regards to RA-ILD, the radiologic findings do appear to correlate reasonably well with the histopathological subtype, and therefore they carry some prognostic significance. 


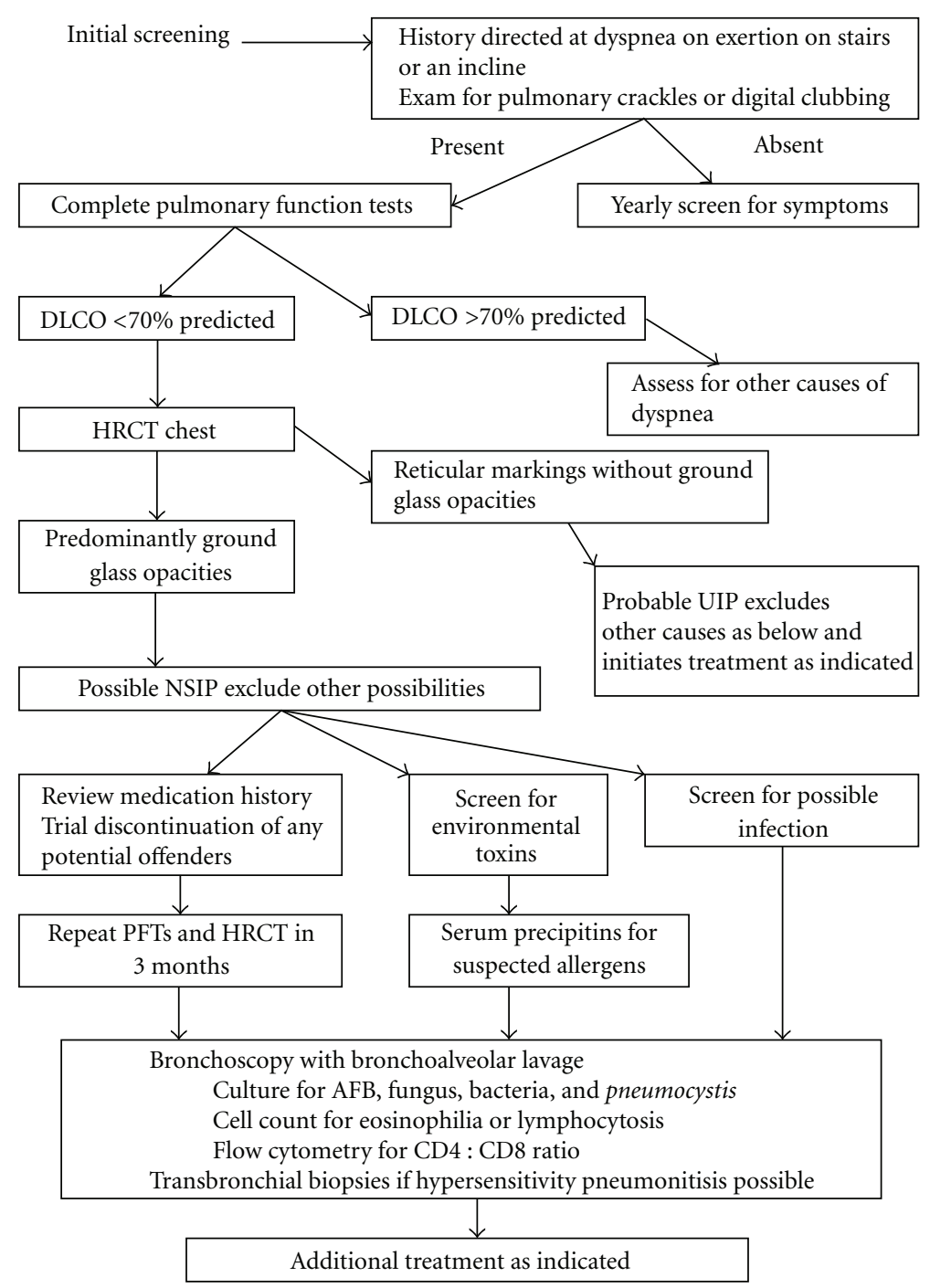

FIGURE 2: Diagnostic algorithm for the evaluation of rheumatoid arthritis.

Usual interstitial pneumonitis (UIP) is the predominant histological finding in RA-ILD affecting more than $50 \%$ of patients $[26,27]$. UIP is the same histological subtype that underlies the end-stage lung disease idiopathic pulmonary fibrosis (IPF), and consequently it carries with it a relatively similar prognosis. It appears that survival is somewhat improved in patients with UIP in RA-ILD as opposed to IPF, but 5 -year survival is estimated to be less than $50 \%$ [28, 29]. In studies comparing HRCT findings with pathological confirmation of UIP, reticular markings and/or peripheral honeycombing are highly sensitive for UIP $[24,26]$. There are a few instances in which HRCT features consistent with UIP were actually found to be fibrotic nonspecific interstitial pneumonitis (fibrotic NSIP) on pathological examination, but it is unclear if this distinction improves the prognosis $[27,30]$. A study by Biederer et al. suggested that men and smokers were more likely to have UIP, with a trend toward purely reticular findings on HRCT as the number of pack years increases [31].
Pathologic findings consistent with NSIP were found to have predominantly ground glass infiltrates on HRCT. This accounts for about a third of the cases of RA-ILD [26]. NSIP is a pattern more often found in women and nonsmokers, and it suggests active alveolar inflammation. Consequently, there is usually a favorable response to immunosuppressive medications, and prognosis is much better with survival estimates greater than $80 \%$ at 5 years from diagnosis [32].

Bronchiolitis obliterans-organizing pneumonia (BOOP) occurs less frequently in about $10 \%$ of patients, and it carries a similarly good prognosis as NSIP [33]. Hallmark features on HRCT include subpleural consolidation with patchy ground glass infiltrates. All of the other ILD histologic subtypes have been reported in RA-ILD including lymphocytic interstitial lung disease (LIP), which may have peribronchiolar or centribular nodules and cystic lesions, as well as the more diffuse diseases including desquamative interstitial pneumonitis (DIP), acute interstitial pneumonitis (AIP), and respiratory bronchiolitis-interstitial lung disease (RB-ILD). 
Open lung biopsy is the only definitive procedure to accurately diagnose an interstitial lung disease, but this is rarely necessary in RA-ILD as suggested by the joint ATS-ERS statement regarding the evaluation of interstitial lung disease in collagen vascular diseases [34]. Additionally, there may be a $20 \%$ increase in mortality if an open lung biopsy is performed in the setting of an acute exacerbation of ILD [35]. We do occasionally employ transbronchial biopsies in conjunction with BAL in an attempt to exclude other coexisting infectious, granulomatous, or eosinophilic diseases, but we never make a determination of the histologic injury of an ILD based on this information. It is not uncommon to obtain a pathologic reading of NSIP although the leading edge of the injury or a biopsy from another lung segment may be more consistent with UIP [27]. In general, we focus on a thorough history to evaluate for other potential causes of the radiologic findings and then proceed with bronchoscopy for BAL with or without transbronchial biopsies to exclude an alternative diagnosis as described above.

\section{Differential Diagnosis in Established Disease}

Once a diagnosis has been rendered, the real challenge begins. As our index case for this paper illustrates, a clinical worsening in the context of established disease presents one of the most difficult aspects of care in RA-ILD. We generally define a clinical worsening as the onset of fever, cough, or new/progressive dyspnea on exertion, and/or a decline in the forced vital capacity (FVC) by $10 \%$ or the DLCO by $15 \%$ [34].

Infection remains the leading cause of death in RA, accounting for $20-30 \%$ of the mortality $[36,37]$. Methotrexate is the most extensively reported medication associated with pulmonary drug toxicity, but almost every medication available for the treatment of RA has been implicated in the development of pneumonitis. An often, overlooked possibility for clinical worsening is environmental or occupational exposures. There are clinical characteristics that increase the likelihood of each of these possibilities in any given patient, and all of these need to be explored before determining that a clinical worsening is attributable to the underlying RA-ILD.

\section{Infectious Complications}

RA patients on immunosuppressive therapy are at an increased risk of developing lower respiratory tract infections, particularly if they have pre-existing lung disease. Many infectious organisms, including mycobacterium avium, mycobacterium tuberculosis, and pneumocystis jirovecii, as well as fungal pathogens can have a radiologic appearance that mimics RA-ILD with increased ground glass and interstitial infiltrates. An additional consideration is that chronic prednisone use increases the risk of hospitalization for pneumonia in RA patients in a dose-dependent fashion with doses greater than $10 \mathrm{mg}$ /day more than doubling the risk [38]. Another study highlighted an increased risk of pneumocystis pneumonia in patients taking as little as $15 \mathrm{mg}$ of prednisone per day for more than 8 weeks suggesting that the use of trimethoprim-sulfamethoxazole as prophylaxis in these patients may be beneficial [39].

It is also important to note that reactivation of tuberculosis in the setting of anti-TNF- $\alpha$ agents can occur and manifest with pulmonary infiltrates, but it appears that extrapulmonary $\mathrm{TB}$ may be more common in this setting occurring in $65 \%$ of cases and manifesting as disseminated or hepatosplenic disease or even TB meningitis [40]. It is possible to develop disseminated tuberculosis on antiTNF- $\alpha$ agents despite a negative PPD in patients already receiving mild immunosuppressive medications. This has prompted the British Thoracic Society to recommend an individual risk-benefit assessment in these patients based on the estimated annual risk of MTB multiplied by a factor of 5 [41]. If disseminated MTB is suspected, sampling multiple sites is recommended since the diagnostic yield from sputum or BAL culture is reported as $70 \%$ or less [42].

Any suspicion of an infectious complication necessitates a prompt and thorough investigation. Fever is a common manifestation of drug-induced pneumonitis, and so it is not definitive evidence of an infectious complication. Immunosuppressive medications may prevent a robust leukocyte response to an infectious organism, so our approach to the patient with pulmonary infiltrates often incorporates early bronchoalveolar lavage in addition to routine blood and urine cultures.

\section{Drug-Induced Pneumonitis}

A number of medications used to treat RA have been associated with the development of pulmonary toxicity. We will briefly describe the main pulmonary toxicities associated with each drug class and explore the risk factors for diffuse pneumonitis in the case of methotrexate and the anti-TNF$\alpha$ biologic agents. Table 2 is provided to summarize these toxicities.

Environmental Toxins. As our index case would suggest, a thorough review of potential environmental toxins needs to be explored. There are over 300 environmental exposures that can increase the odds of developing a hypersensitivity pneumonitis (extrinsic allergic alveolitis) [73]. Specific questions during the clinical history should address possible inhaled allergens in the home or work place including mold, dusts, chemicals, and pet dander. In particular, our patient kept cockatiels as house pets putting her at risk for bird fancier's lung. Other examples include farmer's lung caused by thermophilic actinomycetes, lab worker's lung due to rodent antigens, and epoxy-resin lung due to phtalic anhyhdride [73].

Less than $20 \%$ of people with known exposures actually develop extrinsic allergic alveolitis, but acute symptoms included dyspnea, cough, and malaise and fever in some patients [74]. Chronic exposure can lead to pulmonary fibrosis [74]. HRCT will often demonstrate bilateral ground 
glass opacities and/or centrilobular nodules similar to RAILD [75]. If there is a known exposure, blood tests for serum precipitins to the suspected antigen are indicated. The finding of poorly formed noncaseating granulomas in specimens obtained by transbronchial biopsy can be helpful since these are atypical in RA-ILD as well as MTX pneumonitis where BAL also shows a lymphocytosis similar to hypersensitivity pneumonitis [73]. If a diagnosis of hypersensitivity pneumonitis is rendered, antigen avoidance can be curative, but it often takes up to three weeks to recover from an acute episode, and chronic damage may be irreversible. Steroid therapy may hasten the improvement in symptoms.

Malignancy. The presence of RA-ILD increases the odds of developing lung cancer, but immunosuppression itself can also raise the cancer risk [28]. Diffuse infiltrates may actually represent hematogenous or more likely lymphangitic spread of an underlying malignancy. While this may be a rare complication, it is one that should be considered when a patient seemingly fails to respond to treatment. Additionally, BAL in combination with transbronchial biopsy may provide the correct diagnosis in cases of lymphangitic spread.

7.1. Nonsteroidal Anti-Inflammatory Drugs (NSAIDs). In high doses, NSAIDs have been implicated in the development of diffuse pulmonary infiltrates. In approximately $10-15 \%$ of cases, these infiltrates were simply pulmonary edema, but often these infiltrates reflect an acute eosinophilic pneumonia [43]. In fact, a review by Goodwin and Glenny found naproxen to be the NSAID most commonly associated with acute eosinophilic pneumonia, but there were also cases associated with just any NSAID [44]. Thankfully, the acute inflammation associated with these medications does not typically translate into chronic fibrotic lung disease, and withdrawal of the offending agent with or without additional steroid therapy resolves the inflammatory response.

7.2. Gold Salts and Penicillamine. Gold salts and penicillamine seem to have fallen out of favor with the advent of methotrexate and the newer biologic agents, but a few patients continue to use these medications. The classic pulmonary toxicities associated with these drugs include interstitial pneumonitis, bronchiolitis obliterans with or without organizing pneumonia, pulmonary renal syndromes, and diffuse alveolar damage [45-47].

7.3. Methotrexate. The most frequently implicated RA drug leading to pulmonary toxicity is methotrexate (MTX), but the estimated risk of developing MTX pneumonitis may actually be relatively low at doses of $20-25 \mathrm{mg} /$ week. One study found that only $3 \%$ of 130 patients followed for seven years actually developed MTX-induced pulmonary toxicity [48]. Unfortunately, the subset of patients developing MTX pneumonitis faced a possible $20 \%$ increased mortality rate especially if the drug toxicity occurred in the first six months of treatment [49]. Consequently, attempts to identify patients at risk for pneumonitis should be an important therapeutic goal.

Saravanan and Kelly found that the greatest predictor for the development of pneumonitis is pre-existing pulmonary disease, with a baseline abnormality in the diffusing capacity less than $70 \%$ of predicted incurring a 10 -fold increased risk of MTX drug toxicity [50]. Tobacco abuse, hypoalbuminemia, and previous use of DMARDs including sulfasalazine, gold salts, or D-penicillamine also have large attributable risks [51]. Minor risk factors including advanced age, diabetes mellitus, and RA pleuropulmonary involvement increase the risk for MTX pneumonitis [37, 52, 53]. Our current recommendation is to obtain pulmonary function tests prior to the institution of MTX, and if the DLCO is less than $70 \%$ of predicted, and an HRCT shows any evidence of pleural or interstitial thickening that another agent considered.

If treatment has already commenced, several clinical parameters can be monitored for the development of acute MTX drug toxicity. Surprisingly, routine surveillance of PFTs is not useful in predicting drug toxicity, perhaps due to the acute onset of symptoms [54, 55]. However, duration of therapy has some clinical utility, since roughly half of patients that are going to develop MTX drug toxicity will be symptomatic within the first $6-8$ months of treatment with $10 \%$ of those patients developing symptoms in the first 10 weeks [49]. Low-grade fever is the most prevalent symptom, reported in greater than $90 \%$ of patients. Roughly $80 \%$ of patients also developed a dry, nonproductive cough with the vast majority of these patients also reporting dyspnea on exertion [49].

Fever should prompt a thorough investigation for an infectious etiology, and if bronchoscopy is employed, a bronchoalveolar lavage cell count and flow cytometry can be useful. The lavage fluid will often show a shift away from a neutrophilic cell type to a predominantly lymphocytic infiltrate, with an increased CD4:CD8 ratio [56, 57]. Additionally, a small study by Inokuma et al. revealed a decline in the serum absolute lymphocyte count below 500 cells $/ \mathrm{mm}^{3}$ was closely associated with MTX drug toxicity [58]. Imaging with HRCT characteristically displays a diffuse interstitial pattern in greater than $93 \%$ of patients with MTX pneumonitis. Pleural thickening and less commonly pleural effusions were also found in a small subset of patients [49]. While it is exceedingly unnecessary, an open lung biopsy can help distinguish between RA-ILD and MTX pneumonitis. In general, MTX pneumonitis will have features of acute and organizing diffuse alveolar damage with cellular interstitial infiltrates with or without granulomas [59]. This pattern may also be seen in some infections but generally differs from the classic pathologic features of UIP and NSIP in RA-ILD. However, given the availability of other medications to treat $\mathrm{RA}$, open lung biopsy is rarely necessary.

Two other patterns of chronic injury have been reported as well. A few patients have developed an insidious alveolar fibrosis in the absence of systemic symptoms as well as a persistent chronic cough that were relieved with cessation of MTX therapy $[57,60]$. Additionally, there are several studies that have shown a decrease in the FEV1:FVC ratio and an 
TABLE 2: Common pulmonary drug toxicities and associated risk factors associated with medications used to treat rheumatoid arthritis.

\begin{tabular}{lll}
\hline Medication & Pulmonary toxicity & Risk factors \\
\hline NSAIDS & $\begin{array}{l}\text { Noncardiogenic pulmonary edema, } \\
\text { Acute eosinophilic pneumonia, } \\
\text { Interstitial pneumonitis }\end{array}$ & High-dose treatment \\
\hline Gold salts & $\begin{array}{l}\text { Interstitial pneumoitis, bronchiolitis obliterans } \\
\text { with or without organizing pneumonia, } \\
\text { pulmonary renal syndrome, diffuse alveolar } \\
\text { damage }\end{array}$ & $\begin{array}{l}\text { Unknown, possible genetic association } \\
\text { and cumulative ingestion }>500 \text { mg gold }\end{array}$ \\
\hline D-penicillamine & $\begin{array}{l}\text { Bronchiolitis obliterans, pulmona renal } \\
\text { syndrome, diffuse alveolar damage }\end{array}$ & Unknown \\
\hline Methotrexate & $\begin{array}{l}\text { Acute interstitial pneumonitis, pulmonary } \\
\text { fibrosis, pleural thickening, and chronic cough }\end{array}$ & $\begin{array}{l}\text { DLCO }<70 \% \text { of predicted; tobacco abuse } \\
\text { use of DMARDs; RA pleuropulmonary } \\
\text { involvement; advanced age; diabetes } \\
\text { mellitus }\end{array}$ \\
\hline Anti-TNF- $\alpha$ biologic agents & $\begin{array}{l}\text { Interstitial pneumoitis, rapidly progressive } \\
\text { pulmonary fibrosis }\end{array}$ & Possible prior RA-ILD \\
\hline Leflunomide & Interstitial pneumonitis & $\begin{array}{l}\text { Loading dose of leflunomide; pre-existing } \\
\text { ground glass infiltrates on HRCT }\end{array}$ \\
\hline
\end{tabular}

increased residual volume as the most commonly reported findings on PFTs of patients on chronic MTX therapy [54, $61,62]$.

If acute or chronic toxicity is suspected, withdrawal of MTX is indicated. Once infection has been excluded, treatment of the acute toxicity with supportive care is generally sufficient. In more severe or life-threatening cases, pulse dose glucocorticoids at $1 \mathrm{mg} / \mathrm{kg} /$ day can accelerate the resolution and should be strongly considered [63]. However, if the response to glucocorticoid therapy is inadequate, the addition of azathioprine or cyclophosphamide can dramatically improve the clinical response in some cases $[64,65]$. If a patient develops acute toxicity, rechallenge with MTX is ill advised as the study by Kremer et al. uncovered mortality rates as high as $50 \%$ in this setting [49].

7.4. Leflunomide. Leflunomide was initially reported to cause pneumonitis similar to MTX in Asian populations suggesting a genetic predisposition to pulmonary toxicity [66]. However, case reports from Australia, Germany, and the UK have expanded the awareness of pulmonary toxicity with leflunomide use [67]. In a comprehensive evaluation of the existing literature, pulmonary toxicity occurred within 12 weeks of the initiation of therapy in all patients that received a loading dose of leflunomide and most patients who had pre-existing ILD [67-69]. The mortality associated with the pneumonitis was reported as 19\% predominantly occurring in the subset of patients with previously noted ground glass infiltrates on HRCT [67].

7.5. Anti-TNF- $\alpha$ Biologic Agents. There are case reports of patients initiated on treatment with infliximab, adalimumab, or etanercept who have developed rapidly progressive and sometimes fatal pulmonary fibrosis [70, 71]. Recently, data from the British Society for Rheumatology's Biological
Register (BSRBR) revealed that while the overall mortality was no different between anti-TNF- $\alpha$ agents and DMARDs, mortality from ILD was nearly tripled in patients on antiTNF- $\alpha$ therapy compared to control patients on DMARDs alone [72]. The authors listed some potential problems with the study that may account for that finding, but it appears that pulmonary toxicity associated with these medications is an increasingly recognized problem. More studies will be needed to determine which patients may be at risk for this complication.

\section{Treatment of RA-ILD}

Once a diagnosis of RA-ILD has been made, treatment is generally focused on controlling the systemic disease with immunosuppressive agents while tailoring therapy to the underlying histopathologic subtype. If characteristics of NSIP or BOOP predominate the radiologic appearance with ground glass infiltrates on HRCT, glucocorticoids alone may be effective [76]. We follow a standardized protocol set forth by Lazor et al. in which patients received $0.75 \mathrm{mg} / \mathrm{kg} /$ day prednisone during the initial four weeks of treatment, then $0.5 \mathrm{mg} / \mathrm{kg} /$ day for the next four weeks, then $20 \mathrm{mg} /$ day for four weeks tapering to $10 \mathrm{mg} /$ day for the next 6 weeks, and then $5 \mathrm{mg}$ /day for 6 weeks [77].

A definitive response to treatment is defined as a $10 \%$ improvement in the FVC or $15 \%$ improvement in the DLCO at 12 weeks [32]. Based on recent data from Zappala et al. we continue therapy even if there is a marginal degree of improvement (5-10\% in FVC or $10-15 \%$ in DLCO) [78]. If the patient meets criteria for improvement, we check thiopurine methyltransferase (TPMT) activity levels and if normal initiate steroid sparing therapy with azathioprine $50 \mathrm{mg}$ daily gradually increasing the dose to $2-3 \mathrm{mg} / \mathrm{kg} /$ day [79]. If TPMT activity is decreased, cyclosporine is substituted at a dose of $2.5 \mathrm{mg} / \mathrm{kg} /$ day divided twice daily [80]. If the FVC and DLCO 
remain stable with quarterly PFT assessments over a twoyear period, we consider this evidence that progression of disease has been halted, at which time it may be reasonable to reassess the medical regimen [32].

If there is evidence of reticular markings and honeycombing suggesting UIP, we still attempt a trial of immunosuppressive therapy as described above, but we also treat with $\mathrm{N}$-acetylcysteine $600 \mathrm{mg}$ three times daily based on clinical studies in idiopathic pulmonary fibrosis [81]. Cyclophosphamide $(2 \mathrm{mg} / \mathrm{kg} /$ day orally) is initiated in conjunction with lower-dose prednisone $(0.25 \mathrm{mg} / \mathrm{kg} /$ day $)$ for steroid nonresponders [82]. If they respond, therapy is continued for 18 months to 2 years at which point the medical regimen may be reconsidered or therapy can continue indefinitely. Appropriately selected non-responders are referred for lung transplantation.

One additional challenge involves patients referred for an evaluation of ILD who have occult autoimmune disease. This affects about $10 \%$ of patients who ultimately go on to develop systemic features of rheumatoid arthritis [83]. If the anti-CCP antibodies are positive, we will treat the interstitial lung disease as delineated above and refer to rheumatology for further evaluation and management.

\section{Treatment of an RA-ILD Exacerbation}

An ILD exacerbation is generally defined as rapidly deteriorating respiratory symptoms within a 30-day period with evidence of new infiltrates (usually new ground glass opacities) and exclusion of an identifiable cause [84]. The absolute risk of an acute exacerbation is not well established, but based on the limited data of acute exacerbations in collagen vascular diseases, possibly as many as $20 \%$ of patients with RA-ILD will experience an acute exacerbation with a 1-year incidence as high as $2.58 \%$ [85]. By the time patients present to the hospital, hypoxemia may be severe and a terminal outcome may be eminent $[85,86]$.

Once alternative explanations for a clinical worsening, such as infection and/or drug reaction, have been excluded, treatment is dictated by the underlying histopathology. As common with many rare diseases, there is a paucity of welldesigned clinical trials to guide therapy in RA-ILD, but if the underlying histopathology is NSIP or BOOP, there is a reasonable probability for a sustained clinical response to glucocorticoid treatment alone or in conjunction with other immunosuppressive agents [76]. However, ground glass infiltrates on the background of UIP often herald the development of diffuse alveolar damage, and the ensuing mortality approaches $80-100 \%$ [85-88]. The only evidence to support treatment in this cohort comes from a handful of isolated case reports, while more robust observational studies have demonstrated a consistently poor prognosis [85-88]. Given the lack of evidence to support immunosuppression in UIP, decisions to treat must be made on an individual basis.

We generally initiate treatment with IV methylprednisolone at a dose of $1 \mathrm{mg} / \mathrm{kg} /$ day for 3 days followed by oral prednisolone $1 \mathrm{mg} / \mathrm{kg} /$ day. If there is no evidence of improvement in $\mathrm{paO}_{2} / \mathrm{FiO}_{2}$ ratio or oxygen saturation rescue therapy with cyclophosphamide $\left(500-750 \mathrm{mg} / \mathrm{m}^{2}\right)$ may be considered [89]. Other regimens have employed cyclosporine $3 \mathrm{mg} / \mathrm{kg} /$ day or tacrolimus $3 \mathrm{mg} / \mathrm{kg} /$ day in divided doses in patients unresponsive to cyclophosphamide with some success in isolated case reports $[90,91]$. If the patient progresses to the point of requiring ventilatory support, consideration should be given to noninvasive ventilation, and/or lower PEEP settings and avoidance of recruitment maneuvers, since PEEP levels $>10 \mathrm{~cm} \mathrm{H}_{2} \mathrm{O}$ had more than a 4-fold associated risk of subsequent mortality in one study of acute ILD exacerbations [92].

\section{Other Possible Drug Therapies}

10.1. Anticoagulation. In situ thrombosis within the pulmonary vasculature has been documented in ILD, and a trial of anticoagulation with warfarin by Kubo et al. demonstrated improved survival in this patient population [93]. This may be a reasonable medical therapy in patients with extensive RA-ILD although there are no specific trials investigating its use in this patient population.

10.2. N-Acetylcysteine (NAC). NAC is an antioxidant found to be mildly effective at preventing the decline in lung function in idiopathic pulmonary fibrosis (IPF) [81]. While there is no data regarding its use in RA-ILD, its side effect profile mirrors multivitamins. Since UIP is the predominant histopathology in RA-ILD and is the hallmark pathologic finding in IPF, a dose of $600 \mathrm{mg}$ three times daily may be reasonable to consider in RA-ILD.

10.3. Mycophenolate. This medication has been used for the treatment of RA-ILD and it appears effective in patients with early or limited disease at a dose of 1-2 mg/day [94, 95]. It does not appear to be that effective in relieving articular manifestations of the disease, and therefore it needs to be used in conjunction with DMARD's in most cases.

10.4. Rituximab. This biologic agent is an anti-CD20 antibody licensed for the treatment of RA refractory to antiTNF- $\alpha$ therapy. Interestingly, RA-ILD patients with a UIP histological pattern have increased numbers of CD-20 positive B cells aggregated around small airways [96]. Although there are no published trials on the use of rituximab for RA-ILD, Popa et al. found that RA patients treated with rituximab for 7 years have not shown any evidence of new RA-ILD [97]. There are also several cases reporting the successful use of weekly rituximab for ILD in other forms of collagen vascular disease [98-101].

The optimal treatment strategy remains elusive due to the rare nature of the disease. Hopefully, increased coordination among multiple academic centers will help advance the evaluation of novel medications in large, well-conducted, clinical trials. 


\section{Conclusion}

It is becoming increasingly obvious that RA-ILD is a more prominent and debilitating manifestation of RA than once believed. As our index case demonstrated, there are host of factors that can obscure the diagnosis, but prompt diagnosis and treatment may delay the progression to endstage lung disease. Unfortunately, once RA-ILD has been diagnosed, any acute worsening engages the clinician in an often, challenging evaluation process. Is it the disease, the medications, or some other unrelated but reversible factors contributing to a decline in lung function?

In our patient, reticular markings and ground glass opacities were present in late 2007, but she did not experience significant symptoms for another several months. When she presented for evaluation, she already had a decline in her diffusing capacity to $61 \%$ of predicted. An infectious source was excluded, and it would have been very easy to attribute her symptoms to an obvious case of RA-ILD. However, given her environmental exposures, serum precipitins were drawn to evaluate for a hypersensitivity pneumonitis. She was found to have elevated IgG precipitins to cockatiels, and lymphocytosis was noted on bronchoalveolar lavage. Given a diagnosis of probable hypersensitivity pneumonitis she was started on high-dose prednisone, and she removed her cockatiels from the home. Over the next three months her DLCO improved to $89 \%$. Her prednisone was tapered to $10 \mathrm{mg}$ daily, and one year later her, DLCO had been normalized at $98 \%$ of predicted.

\section{References}

[1] M. Fujii, S. Adachi, T. Shimizu, S. Hiroto, M. Sako, and M. Kono, "Interstitial lung disease in rheumatoid arthritis: assessment with high-resolution computed tomography," Journal of Thoracic Imaging, vol. 8, no. 1, pp. 54-62, 1993.

[2] E. Gabbay, R. Tarala, R. Will et al., "Interstitial lung disease in recent onset rheumatoid arthritis," American Journal of Respiratory and Critical Care Medicine, vol. 156, no. 2, part 1, pp. 528-535, 1997.

[3] H. Toyoshima, T. Kusaba, and M. Yamaguchi, "Cause of death in autopsied RA patients," Ryumachi, vol. 33, no. 3, pp. 209214, 1993.

[4] J. McDonagh, M. Greaves, A. R. Wright, C. Heycock, J. P. Owen, and C. Kelly, "High resolution computed tomography of the lungs in patients with rheumatoid arthritis and interstitial lung disease," British Journal of Rheumatology, vol. 33, no. 2, pp. 118-122, 1994.

[5] B. R. Gochuico, N. A. Avila, C. K. Chow et al., "Progressive preclinical interstitial lung disease in rheumatoid arthritis," Archives of Internal Medicine, vol. 168, no. 2, pp. 159-166, 2008.

[6] S. C. Morrison, G. M. Mody, S. R. Benatar, and O. L. Meyers, "The lungs in rheumatoid arthritis-a clinical, radiographic and pulmonary function study," South African Medical Journal, vol. 86, no. 7, pp. 829-833, 1996.

[7] W. C. Walker and V. Wright, "Pulmonary lesions and rheumatoid arthritis," Medicine, vol. 47, no. 6, pp. 501-520, 1968.

[8] P. Cervantes-Perez, A. H. Toro-Perez, and P. RodriguezJurado, "Pulmonary involvement in rheumatoid arthritis,"
Journal of the American Medical Association, vol. 243, no. 17, pp. 1715-1719, 1980.

[9] B. Cortet, R. M. Flipo, M. Remy-Jardin et al., "Use of high resolution computed tomography of the lungs in patients with rheumatoid arthritis," Annals of the Rheumatic Diseases, vol. 54, no. 10, pp. 815-819, 1995.

[10] J. K. Dawson, H. E. Fewins, J. Desmond, M. P. Lynch, and D. R. Graham, "Fibrosing alveolitis in patients with rheumatoid arthritis as assessed by high resolution computed tomography, chest radiography, and pulmonary function tests," Thorax, vol. 56, no. 8, pp. 622-627, 2001.

[11] D. M. Geddes, M. Webley, and P. A. Emerson, "Airways obstruction in rheumatoid arthritis," Annals of the Rheumatic Diseases, vol. 38, no. 3, pp. 222-225, 1979.

[12] A. Vergnenègre, N. Pugnere, M. T. Antonini et al., "Airway obstruction and rheumatoid arthritis," European Respiratory Journal, vol. 10, no. 5, pp. 1072-1078, 1997.

[13] R. H. Lofgren and W. W. Montgomery, "Incidence of laryngeal involvement in rheumatoid arthritis," The New England Journal of Medicine, vol. 267, pp. 193-195, 1962.

[14] V. Cottin, H. Nunes, P. Y. Brillet et al., "Combined pulmonary fibrosis and emphysema: a distinct underrecognised entity," European Respiratory Journal, vol. 26, no. 4, pp. 586-593, 2005.

[15] J. F. Aduen, D. A. Zisman, S. I. Mobin et al., "Retrospective study of pulmonary function tests in patients presenting with isolated reduction in single-breath diffusion capacity: implications for the diagnosis of combined obstructive and restrictive lung disease," Mayo Clinic Proceedings, vol. 82, no. 1, pp. 48-54, 2007.

[16] S. L. Schmidt et al., "Pulmonary function measures predict mortality differently in idiopathic pulmonary fibrosis versus combined pulmonary fibrosis and emphysema," European Respiratory Journal. In press.

[17] S. A. Albano, E. Santana-Sahagun, and M. H. Weisman, "Cigarette smoking and rheumatoid arthritis," Seminars in Arthritis and Rheumatism, vol. 31, no. 3, pp. 146-159, 2001.

[18] E. J. Kim, H. R. Collard, and T. E. King Jr., "Rheumatoid arthritis-associated interstitial lung disease: the relevance of histopathologic and radiographic pattern," Chest, vol. 136, no. 5, pp. 1397-1405, 2009.

[19] D. E. O’Donnell, J. Ora, K. A. Webb, P. Laveneziana, and D. Jensen, "Mechanisms of activity-related dyspnea in pulmonary diseases," Respiratory Physiology and Neurobiology, vol. 167, no. 1, pp. 116-132, 2009.

[20] C. M. Weyand, D. Schmidt, U. Wagner, and J. J. Goronzy, "The influence of sex on the phenotype of rheumatoid arthritis," Arthritis and Rheumatism, vol. 41, no. 5, pp. 817822, 1998.

[21] K. G. Saag, S. Kolluri, R. K. Koehnke et al., "Rheumatoid arthritis lung disease: determinants of radiographic and physiologic abnormalities," Arthritis and Rheumatism, vol. 39, no. 10, pp. 1711-1719, 1996.

[22] I. Alexiou, A. Germenis, A. Koutroumpas, A. Kontogianni, K. Theodoridou, and L. I. Sakkas, "Anti-cyclic citrullinated peptide-2 (CCP2) autoantibodies and extra-articular manifestations in Greek patients with rheumatoid arthritis," Clinical Rheumatology, vol. 27, no. 4, pp. 511-513, 2008.

[23] N. Tanaka, J. S. Kim, J. D. Newell et al., "Rheumatoid arthritis-related lung diseases: CT findings," Radiology, vol. 232, no. 1, pp. 81-91, 2004.

[24] M. Akira, M. Sakatani, and H. Hara, "Thin-section CT findings in rheumatoid arthritis-associated lung disease: CT 
patterns and their courses," Journal of Computer Assisted Tomography, vol. 23, no. 6, pp. 941-948, 1999.

[25] E. S. White, H. D. Tazelaar, and J. P. Lynch III, "Bronchiolar complications of connective tissue diseases," Seminars in Respiratory and Critical Care Medicine, vol. 24, no. 5, pp. 543565, 2003.

[26] H. K. Lee, D. S. Kim, B. Yoo et al., "Histopathologic pattern and clinical features of rheumatoid arthritis-associated interstitial lung disease," Chest, vol. 127, no. 6, pp. 2019-2027, 2005.

[27] D. Tansey, A. U. Wells, T. V. Colby et al., "Variations in histological patterns of interstitial pneumonia between connective tissue disorders and their relationship to prognosis," Histopathology, vol. 44, no. 6, pp. 585-596, 2004.

[28] A. Rajasekaran, D. Shovlin, V. Saravanan, P. Lord, and C. Kelly, "Interstitial lung disease in patients with rheumatoid arthritis: comparison with cryptogenic fibrosing alveolitis over 5 years," Journal of Rheumatology, vol. 33, no. 7, pp. 1250-1253, 2006.

[29] J. H. Park, D. S. Kim, I. N. Park et al., "Prognosis of fibrotic interstitial pneumonia: idiopathic versus collagen vascular disease-related subtypes," American Journal of Respiratory and Critical Care Medicine, vol. 175, no. 7, pp. 705-711, 2007.

[30] W. D. Travis, K. Matsui, J. Moss, and V. J. Ferrans, "Idiopathic nonspecific interstitial pneumonia: prognostic significance of cellular and fibrosing patterns — survival comparison with usual interstitial pneumonia and desquamative interstitial pneumonia," American Journal of Surgical Pathology, vol. 24, no. 1, pp. 19-33, 2000.

[31] J. Biederer, A. Schnabel, C. Muhle, W. L. Gross, M. Heller, and M. Reuter, "Correlation between HRCT findings, pulmonary function tests and bronchoalveolar lavage cytology in interstitial lung disease associated with rheumatoid arthritis," European Radiology, vol. 14, no. 2, pp. 272-280, 2004.

[32] W. D. Travis, G. Hunninghake, T. E. King et al., "Idiopathic nonspecific interstitial pneumonia: report of an American Thoracic Society Project," American Journal of Respiratory and Critical Care Medicine, vol. 177, no. 12, pp. 1338-1347, 2008.

[33] H. Hayakawa, A. Sato, S. Imokawa, M. Toyoshima, K. Chida, and M. Iwata, "Bronchiolar disease in rheumatoid arthritis," American Journal of Respiratory and Critical Care Medicine, vol. 154, no. 5, pp. 1531-1536, 1996.

[34] American Thoracic Society (ATS), and the European Respiratory Society (ERS), "American thoracic society/European respiratory society international multidisciplinary consensus classification of the idiopathic interstitial pneumonias," American Journal of Respiratory and Critical Care Medicine, vol. 165, no. 2, pp. 277-304, 2002.

[35] J. H. Park, D. K. Kim, D. S. Kim et al., "Mortality and risk factors for surgical lung biopsy in patients with idiopathic interstitial pneumonia," European Journal of Cardio-thoracic Surgery, vol. 31, no. 6, pp. 1115-1119, 2007.

[36] C. Turesson, L. Jacobsson, and U. Bergström, "Extra-articular rheumatoid arthritis: prevalence and mortality," Rheumatology, vol. 38, no. 7, pp. 668-674, 1999.

[37] A. Suzuki, Y. Ohosone, M. Obana et al., "Cause of death in 81 autopsied patients with rheumatoid arthritis," Journal of Rheumatology, vol. 21, no. 1, pp. 33-36, 1994.

[38] F. Wolfe, L. Caplan, and K. Michaud, "Treatment for rheumatoid arthritis and the risk of hospitalization for pneumonia: associations with prednisone, diseasemodifying antirheumatic drugs, and anti-tumor necrosis factor therapy," Arthritis and Rheumatism, vol. 54, no. 2, pp. 628-634, 2006.

[39] S. H. Yale and A. H. Limper, "Pneumocystis carinii pneumonia in patients without acquired immunodeficiency syndrome: associated illnesses and prior corticosteroid therapy," Mayo Clinic Proceedings, vol. 71, no. 1, pp. 5-13, 1996.

[40] J. J. Gómez-Reino, L. Carmona, V. Rodríguez Valverde, E. M. Mola, and M. D. Montero, "Treatment of rheumatoid arthritis with tumor necrosis factor inhibitors may predispose to significant increase in tuberculosis risk: a multicenter active-surveillance report," Arthritis and Rheumatism, vol. 48, no. 8, pp. 2122-2127, 2003.

[41] L. P. Ormerod, H. J. Milburn, S. Gillespie, J. Ledingham, and D. Rampton, "BTS recommendations for assessing risk and for managing Mycobacterium tuberculosis infection and disease in patients due to start anti-TNF- $\alpha$ treatment," Thorax, vol. 60, no. 10, pp. 800-805, 2005.

[42] G. Maartens, P. A. Willcox, and S. R. Benatar, "Miliary tuberculosis: rapid diagnosis, hematologic abnormalities, and outcome in 109 treated adults," American Journal of Medicine, vol. 89, no. 3, pp. 291-296, 1990.

[43] J. E. Heffner and S. A. Sahn, "Salicylate-induced pulmonary edema. Clinical features and prognosis," Annals of Internal Medicine, vol. 95, no. 4, pp. 405-409, 1981.

[44] S. D. Goodwin and R. W. Glenny, "Nonsteroidal antiinflammatory drug-associated pulmonary infiltrates with eosinophilia: review of the literature and Food and Drug Administration Adverse Drug Reaction reports," Archives of Internal Medicine, vol. 152, no. 7, pp. 1521-1524, 1992.

[45] M. Turner-Warwick, "Adverse reactions affecting the lung: possible association with D-penicillamine," Journal of Rheumatology, vol. 8, no. 7, pp. 166-168, 1981.

[46] C. J. Eastmond, "Diffuse alveolitis as complication of penicillamine treatment for rheumatoid arthritis," British Medical Journal, vol. 1, no. 6024, p. 1506, 1976.

[47] H. Tomioka and T. E. King Jr., "Gold-induced pulmonary disease: clinical features, outcome, and differentiation from rheumatoid lung disease," American Journal of Respiratory and Critical Care Medicine, vol. 155, no. 3, pp. 1011-1020, 1997.

[48] P. Hilliquin, M. Renoux, S. Perrot, X. Puéchal, and C. J. Menkès, "Occurrence of pulmonary complications during methotrexate therapy in rheumatoid arthritis," British Journal of Rheumatology, vol. 35, no. 5, pp. 441-445, 1996.

[49] J. M. Kremer, G. S. Alarcón, M. E. Weinblatt et al., "Clinical, laboratory, radiographic, and histopathologic features of methotrexate-associated lung injury in patients with rheumatoid arthritis: a multicenter study with literature review," Arthritis and Rheumatism, vol. 40, no. 10, pp. 1829-1837, 1997.

[50] V. Saravanan and C. A. Kelly, "Reducing the risk of methotrexate pneumonitis in rheumatoid arthritis," Rheumatology, vol. 43, no. 2, pp. 143-147, 2004.

[51] G. S. Alarcón, J. M. Kremer, M. Macaluso et al., "Risk factors for methotrexate-induced lung injury in patients with rheumatoid arthritis: a multicenter, case-control study," Annals of Internal Medicine, vol. 127, no. 5, pp. 356-364, 1997.

[52] G. J. Carroll, R. Thomas, C. C. Phatouros et al., "Incidence, prevalence and possible risk factors for pneumonitis in patients with rheumatoid arthritis receiving methotrexate," Journal of Rheumatology, vol. 21, no. 1, pp. 51-54, 1994. 
[53] M. R. Golden, R. S. Katz, R. A. Balk, and H. E. Golden, "The relationship of preexisting lung disease to the development of methotrexate pneumonitis in patients with rheumatoid arthritis," Journal of Rheumatology, vol. 22, no. 6, pp. 1043-1047, 1995.

[54] C. Beyeler, B. Jordi, N. J. Gerber, and V. Im Hof, "Pulmonary function in rheumatoid arthritis treated with low-dose methotrexate: a longitudinal study," British Journal of Rheumatology, vol. 35, no. 5, pp. 446-452, 1996.

[55] V. Cottin, J. Tébib, B. Massonnet, P. J. Souquet, and J. P. Bernard, "Pulmonary function in patients receiving longterm low-dose methotrexate," Chest, vol. 109, no. 4, pp. 933-938, 1996.

[56] C. Fuhrman, A. Parrot, M. Wislez et al., "Spectrum of CD4 to CD8 T-cell ratios in lymphocytic alveolitis associated with methotrexate-induced pneumonitis," American Journal of Respiratory and Critical Care Medicine, vol. 164, no. 7, pp. 1186-1191, 2001.

[57] A. Schnabel, K. Dalhoff, S. Bauerfeind, J. Barth, and W. L. Gross, "Sustained cough in methotrexate therapy for rheumatoid arthritis," Clinical Rheumatology, vol. 15, no. 3, pp. 277-282, 1996.

[58] S. Inokuma, H. Kono, Y. Kohno et al., "Methotrexateinduced lung injury in patients with rheumatoid arthritis occurs with peripheral blood lymphocyte count decrease," Annals of the Rheumatic Diseases, vol. 65, no. 8, pp. 11131114, 2006.

[59] S. Imokawa, T. V. Colby, K. O. Leslie, and R. A. Helmers, "Methotrexate pneumonitis: review of the literature and histopathological findings in nine patients," European Respiratory Journal, vol. 15, no. 2, pp. 373-381, 2000.

[60] F. Salaffi, P. Manganelli, M. Carotti, S. Subiaco, G. Lamanna, and C. Cervini, "Methotrexate-induced pneumonitis in patients with rheumatoid arthritis and psoriatic arthritis: report of five cases and review of the literature," Clinical Rheumatology, vol. 16, no. 3, pp. 296-304, 1997.

[61] C. S. Dayton, D. A. Schwartz, N. L. Sprince et al., "Lowdose methotrexate may cause air trapping in patients with rheumatoid arthritis," American Journal of Respiratory and Critical Care Medicine, vol. 151, no. 4, pp. 1189-1193, 1995.

[62] J. K. Dawson, D. R. Graham, J. Desmond, H. E. Fewins, and M. P. Lynch, "Investigation of the chronic pulmonary effects of low-dose oral methotrexate in patients with rheumatoid arthritis: a prospective study incorporating HRCT scanning and pulmonary function tests," Rheumatology, vol. 41, no. 3, pp. 262-267, 2002.

[63] D. A. Zisman, W. J. McCune, G. Tino, and J. P. Lynch, "Drug-induced pneumonitis: the role of methotrexate," Sarcoidosis Vasculitis and Diffuse Lung Diseases, vol. 18, no. 3, pp. 243-252, 2001.

[64] D. Libby and D. A. White, "Pulmonary toxicity of drugs used to treat systemic autoimmune diseases," Clinics in Chest Medicine, vol. 19, no. 4, pp. 809-821, 1998.

[65] A. Suwa, M. Hirakata, S. Satoh, T. Mimori, K. Utsumi, and S. Inada, "Rheumatoid arthritis associated with methotrexate-induced pneumonitis: improvement with i.v. cyclophosphamide therapy," Clinical and Experimental Rheumatology, vol. 17, no. 3, pp. 355-358, 1999.

[66] Y. Kamata, H. Nara, T. Kamimura et al., "Rheumatoid arthritis complicated with acute interstitial pneumonia induced by leflunomide as an adverse reaction," Internal Medicine, vol. 43, no. 12, pp. 1201-1204, 2004.
[67] B. Chikura, S. Lane, and J. K. Dawson, "Clinical expression of leflunomide-induced pneumonitis," Rheumatology, vol. 48, no. 9, pp. 1065-1068, 2009.

[68] S. Suissa, M. Hudson, and P. Ernst, "Leflunomide use and the risk of interstitial lung disease in rheumatoid arthritis," Arthritis and Rheumatism, vol. 54, no. 5, pp. 1435-1439, 2006.

[69] T. Sawada, S. Inokuma, T. Sato et al., "Leflunomide-induced interstitial lung disease: prevalence and risk factors in Japanese patients with rheumatoid arthritis," Rheumatology, vol. 48, no. 9, pp. 1069-1072, 2009.

[70] A. J. K. Ostor, A. J. Crisp, M. F. Somerville, and D. G. I. Scott, "Fatal exacerbation of rheumatoid arthritis associated fibrosing alveolitis in patients given infliximab," British Medical Journal, vol. 329, no. 7477, p. 1266, 2004.

[71] K. Hagiwara, T. Sato, S. Takagi-Kobayashi, S. Hasegawa, N. Shigihara, and O. Akiyama, "Acute exacerbation of preexisting interstitial lung disease after administration of etanercept for rheumatoid arthritis," Journal of Rheumatology, vol. 34, no. 5, pp. 1151-1154, 2007.

[72] W. G. Dixon, K. L. Hyrich, K. D. Watson, M. Lunt, and D. P. M. Symmons, "Influence of anti-TNF therapy on mortality in patients with rheumatoid arthritis-associated interstitial lung disease: results from the British Society for Rheumatology Biologics Register," Annals of the Rheumatic Diseases, vol. 69, no. 6, pp. 1086-1091, 2010.

[73] M. Selman, "Hypersensitivity pneumonitis: a multifaceted deceiving disorder," Clinics in Chest Medicine, vol. 25, no. 3, pp. 531-547, 2004.

[74] E. S. Yi, "Hypersensitivity pneumonitis," Critical Reviews in Clinical Laboratory Sciences, vol. 39, no. 6, pp. 581-629, 2002.

[75] C. S. Glazer, C. S. Rose, and D. A. Lynch, "Clinical and radiologic manifestations of hypersensitivity pneumonitis," Journal of Thoracic Imaging, vol. 17, no. 4, pp. 261-272, 2002.

[76] R. A. Roschmann and R. J. Rothenberg, "Pulmonary fibrosis in rheumatoid arthritis: a review of clinical features and therapy," Seminars in Arthritis and Rheumatism, vol. 16, no. 3, pp. 174-185, 1987.

[77] R. Lazor, A. Vandevenne, A. Pelletier et al., "Cryptogenic organizing pneumonia: characteristics of relapses in a series of 48 patients," American Journal of Respiratory and Critical Care Medicine, vol. 162, no. 2, part 1, pp. 571-577, 2000.

[78] C. J. Zappala, P. I. Latsi, A. G. Nicholson et al., "Marginal decline in forced vital capacity is associated with a poor outcome in idiopathic pulmonary fibrosis," European Respiratory Journal, vol. 35, no. 4, pp. 830-835, 2010.

[79] R. W. Simms, C. K. Kwoh, L. G. Anderson et al., "Guidelines for monitoring drug therapy in rheumatoid arthritis: American College of Rheumatology Ad Hoc Committee on Clinical Guidelines," Arthritis and Rheumatism, vol. 39, no. 5, pp. 723-731, 1996.

[80] M. P. E. Puttick, A. V. Klinkhoff, A. Chalmers, and D. N. Ostrow, "Treatment of progressive rheumatoid interstitial lung disease with cyclosporine," Journal of Rheumatology, vol. 22, no. 11, pp. 2163-2165, 1995.

[81] M. Demedts, J. Behr, R. Buhl et al., "High-dose acetylcysteine in idiopathic pulmonary fibrosis," New England Journal of Medicine, vol. 353, no. 21, pp. 2229-2242, 2005.

[82] N. Nanki, J. Fujita, Y. Yamaji et al., "Nonspecific interstitial pneumonia/fibrosis completely recovered by adding cyclophosphamide to corticosteroids," Internal Medicine, vol. 41, no. 10, pp. 867-870, 2002. 
[83] G. E. Tzelepis, S. P. Toya, and H. M. Moutsopoulos, "Occult connective tissue diseases mimicking idiopathic interstitial pneumonias," European Respiratory Journal, vol. 31, no. 1, pp. 11-20, 2008.

[84] H. R. Collard, B. B. Moore, K. R. Flaherty et al., "Acute exacerbations of idiopathic pulmonary fibrosis," American Journal of Respiratory and Critical Care Medicine, vol. 176, no. 7, pp. 636-643, 2007.

[85] T. Suda, Y. Kaida, Y. Nakamura et al., "Acute exacerbation of interstitial pneumonia associated with collagen vascular diseases," Respiratory Medicine, vol. 103, no. 6, pp. 846-853, 2009.

[86] S. A. Papiris et al., "Clinical review: idiopathic pulmonary fibrosis acute exacerbations-unravelling Ariadne's thread," Critical Care, vol. 14, no. 6, p. 246, 2010.

[87] I. N. Park, D. S. Kim, T. S. Shim et al., "Acute exacerbation of interstitial pneumonia other than idiopathic pulmonary fibrosis," Chest, vol. 132, no. 1, pp. 214-220, 2007.

[88] J. A. Bjoraker, J. H. Ryu, M. K. Edwin et al., "Prognostic significance of histopathologic subsets in idiopathic pulmonary fibrosis," American Journal of Respiratory and Critical Care Medicine, vol. 157, no. 1, pp. 199-203, 1998.

[89] S. Mori et al., "Idiopathic interstitial pneumonia that responded to intermittent intravenous administration of high-dose cyclophosphamide," Nihon Kyobu Shikkan Gakkai Zasshi, vol. 32, no. 10, pp. 983-989, 1994.

[90] T. Naniwa, H. Tomita, S. Ninomiya et al., "Successful use of immunosuppressive agents for the treatment of progressive rheumatoid interstitial lung disease: a case report," Nihon Kokyuki Gakkai Zasshi, vol. 40, no. 1, pp. 55-60, 2002.

[91] S. Ochi, T. Kubota, T. Sugihara et al., "A case report of rheumatoid arthritis complicated with rapidly progressive interstitial pneumonia, multiple bullae and pneumomediastinum, which was successfully treated with tacrolimus," Nihon Rinsho Meneki Gakkai Kaishi, vol. 31, no. 1, pp. 62-67, 2008.

[92] E. R. Fernández-Pérez, M. Yilmaz, H. Jenad et al., "Ventilator settings and outcome of respiratory failure in chronic interstitial lung disease," Chest, vol. 133, no. 5, pp. 11131119, 2008.

[93] H. Kubo, K. Nakayama, M. Yanai et al., "Anticoagulant therapy for idiopathic pulmonary fibrosis," Chest, vol. 128, no. 3, pp. 1475-1482, 2005.

[94] J. J. Swigris, A. L. Olson, A. Fischer et al., "Mycophenolate mofetil is safe, well tolerated, and preserves lung function in patients with connective tissue disease-related interstitial lung disease," Chest, vol. 130, no. 1, pp. 30-36, 2006.

[95] L. A. Saketkoo and L. R. Espinoza, "Rheumatoid arthritis interstitial lung disease: mycophenolate mofetil as an antifibrotic and disease-modifying antirheumatic drug," Archives of Internal Medicine, vol. 168, no. 15, pp. 1718-1719, 2008.

[96] S. R. Atkins, C. Turesson, J. L. Myers et al., "Morphologic and quantitative assessment of $\mathrm{CD} 20+\mathrm{B}$ cell infiltrates in rheumatoid arthritis-associated nonspecific interstitial pneumonia and usual interstitial pneumonia," Arthritis and Rheumatism, vol. 54, no. 2, pp. 635-641, 2006.

[97] C. Popa, M. J. Leandro, G. Cambridge, and J. C. W. Edwards, "Repeated B lymphocyte depletion with rituximab in rheumatoid arthritis over 7 yrs," Rheumatology, vol. 46, no. 4, pp. 626-630, 2007.

[98] S. W. Lim, D. Gillis, W. Smith, P. Hissaria, H. Greville, and C. A. Peh, "Rituximab use in systemic lupus erythematosus pneumonitis and a review of current reports," Internal Medicine Journal, vol. 36, no. 4, pp. 260-262, 2006.

[99] O. Lambotte, R. Kotb, G. Maigne, F. X. Blanc, C. Goujard, and J. F. Delfraissy, "Efficacy of rituximab in refractory polymyositis," Journal of Rheumatology, vol. 32, no. 7, pp. 1369-1370, 2005.

[100] K. A. Keogh, S. R. Ytterberg, F. C. Fervenza, K. A. Carlson, D. R. Schroeder, and U. Specks, "Rituximab for refractory Wegener's granulomatosis: report of a prospective, openlabel pilot trial," American Journal of Respiratory and Critical Care Medicine, vol. 173, no. 2, pp. 180-187, 2006.

[101] M. Koukoulaki, K. G. C. Smith, and D. R. W. Jayne, "Rituximab in Churg-Strauss syndrome," Annals of the Rheumatic Diseases, vol. 65, no. 4, pp. 557-559, 2006. 


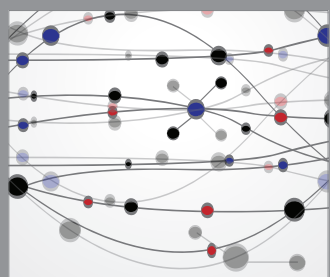

The Scientific World Journal
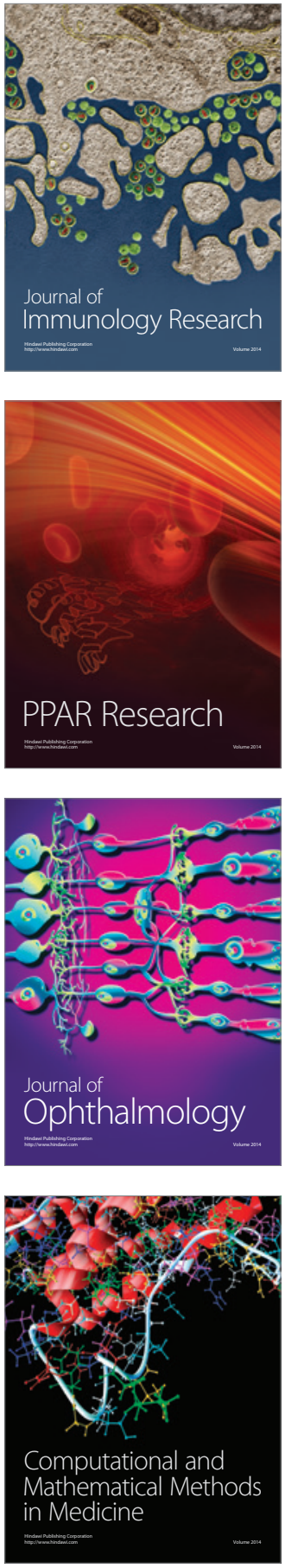

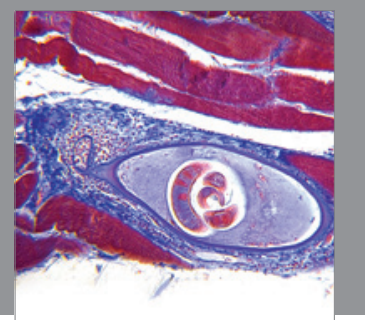

Gastroenterology

Research and Practice
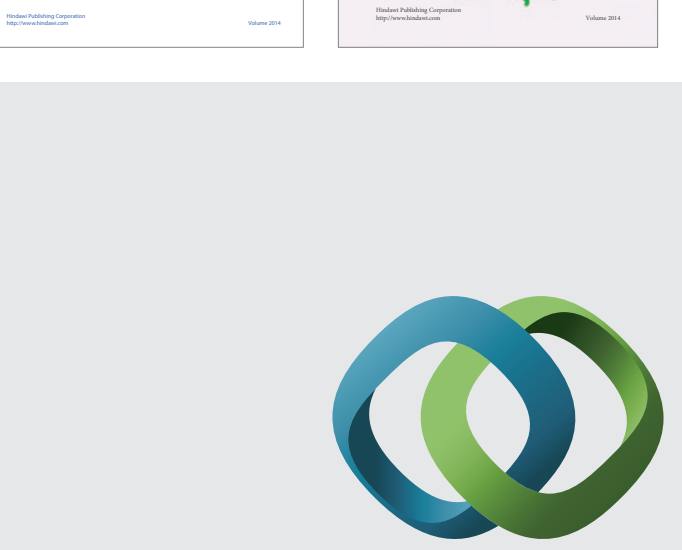

\section{Hindawi}

Submit your manuscripts at

http://www.hindawi.com
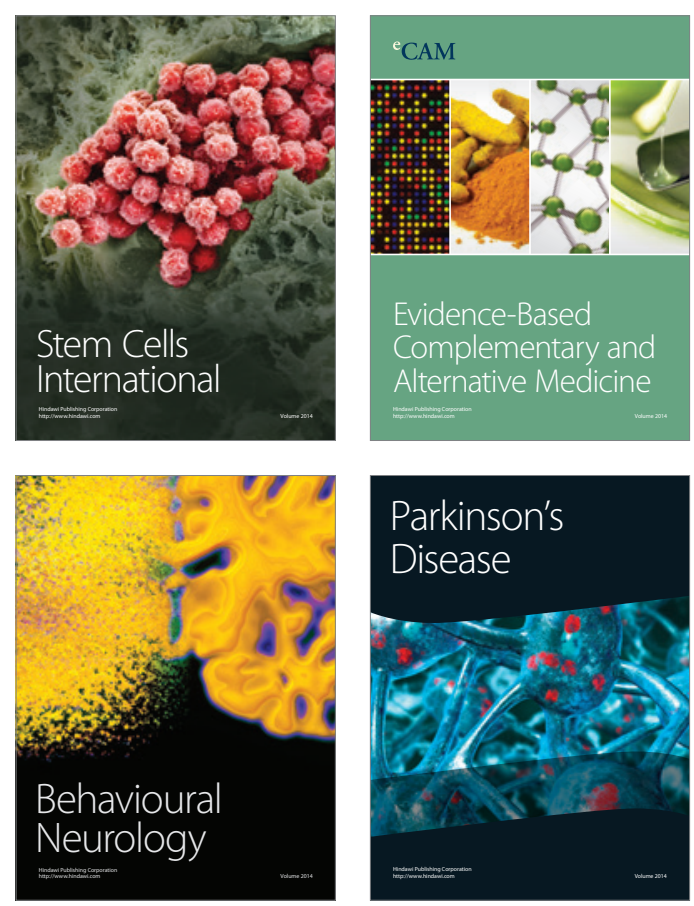

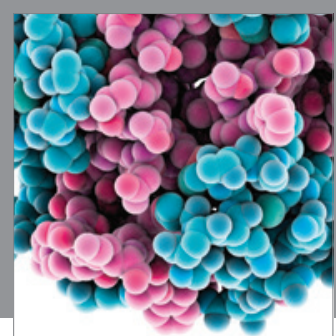

Journal of
Diabetes Research

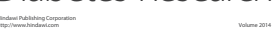

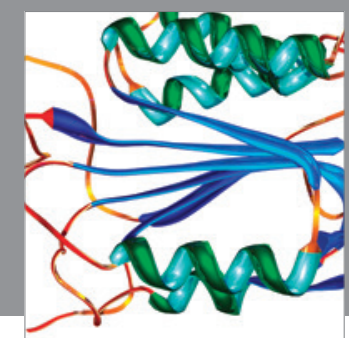

Disease Markers
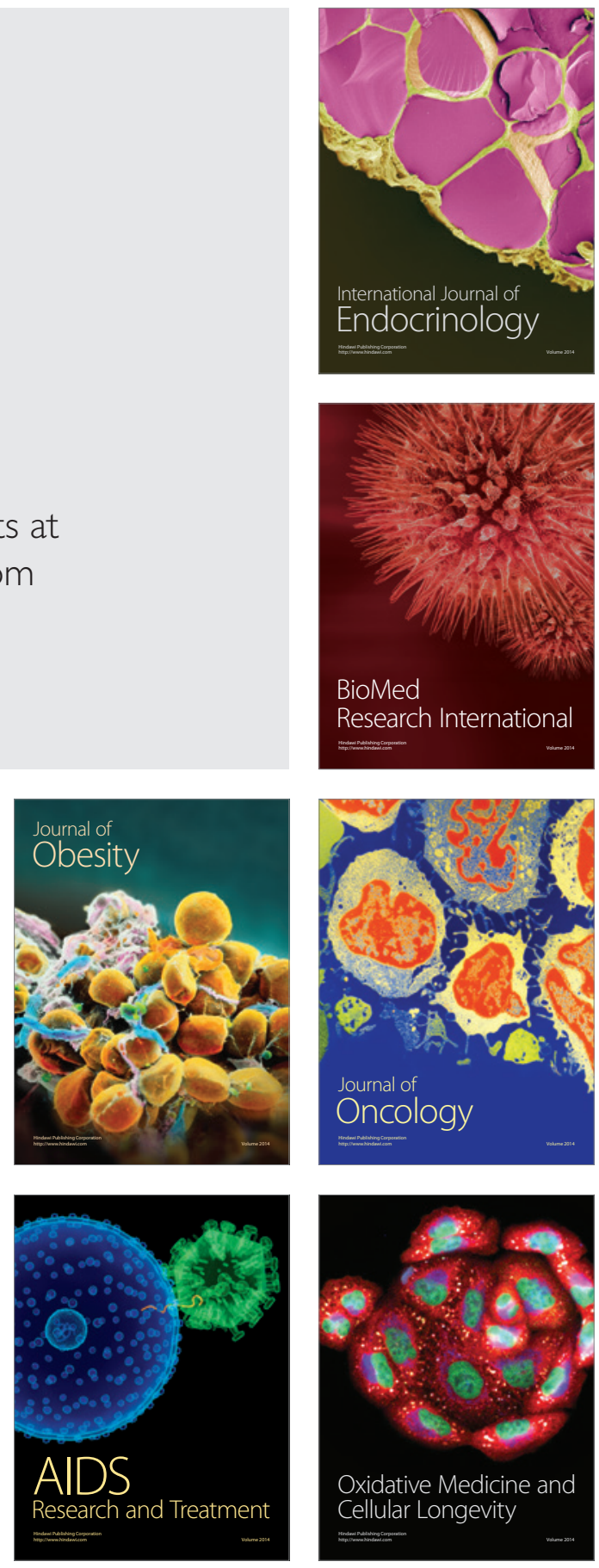